\title{
EVALUATION OF OPTIMUM CONDITIONS FOR SOIL TERMITICIDES STORAGE IN TROPICAL CLIMATE
}

\author{
Mohd Fawwaz Mohd Rashid ${ }^{1 a}$ and Abdul Hafiz Ab Majid ${ }^{2 a^{*}}$ \\ ${ }^{a}$ Household and Structural Urban Entomology Laboratoty, Vector Control Research Unit, School of Biological \\ Sciences, Universiti Sains Malaysia, 11800 Minden, Penang, MALAYSIA. Email: fawwaz2907@gmail.com ${ }^{1}$; \\ abdhafiz@usm.my² \\ Corresponding author: abdhafiz@usm.my \\ Received: $7^{\text {th }}$ May 2019 \\ Accepted: $24^{\text {th }}$ Jan 2020 \\ Published: $29^{\text {th }}$ Feb 2020
}

DOI: https://doi.org/10.22452/mjs.vol39no1.7

\begin{abstract}
Soil termiticide treatment is a key strategy in controlling the termite population and infestation by creating a continuous barrier encompassing the structures. Termiticides, however, may dissipate for a variety of reasons such as a half-life, a degradation rate, a leaching activity, and a storage method. This study aimed to evaluate the optimum storage conditions for three of the most commonly used termiticides i.e. bifenthrin, fipronil, and imidacloprid. The evaluation was made by examining the Active Ingredients (AIs) of each termiticide using two sets of colored bottles (black and white) and two different storage areas (in car and store) within 30 days. The results revealed that the colored bottles $(\mathrm{F}: 0.181, \mathrm{df}=1, \mathrm{P}=$ $0.672)$ and storage areas $(\mathrm{F}=5.495, \mathrm{df}=1, \mathrm{P}=0.977)$ did not significantly affect the concentration of termiticides after 30 days. It further demonstrated that the tested termiticides were unstable in an aqueous solution after 30 days due to hydrolysis. The half-life values were recorded at 7.45 days (bifenthrin), 3.98 days (fipronil) and 9.42 days (imidacloprid), respectively. The black and white bottles showed no significant effect on termiticide concentrations, indicating that photodegradation could occur regardless if the termiticides were stored in the building or in the car. Fipronil was the least effective termiticide as its residual reduced to half after three days of mixing. Hence, it is recommended that any termiticide must be immediately used after mixing to avoid photodegradation and hydrolysis.
\end{abstract}

Keywords: Termiticides, storage, degradation, half-life, aqueous

\section{INTRODUCTION}

For decades, the application of termiticides is the common practice in termite control management. However, excessive and prolonged use of termiticides as the control measure has led to staggering impacts on humans, fishes, livestock and wildlife animals (Floate et al., 2005; Joshi et al., 2016; Petroff 2010; Phung et al., 2012). The adverse effect of overly used termiticides further creates widespread worries among environmentalists. A proper storing procedure is thus, needed to lessen the harmful risk and to sustain the effectiveness of termiticides. The purpose of storing termiticides are varied, for instance, to prevent termiticide from spilling (Petroff, 2010), water contamination (Transporting and Storing Pesticides Safely, 2016), and degradation (Husen et al., 2009).

Pesticide Act (1974) Section 20 (2) strictly prohibits the selling or storage of the 
pesticides at a place other than the specific premises with good conditions in order to maintain the quality of the pesticides from degradation. Among others, preparing a suitable condition for storing termiticides prior to mixing and the fate of unused termiticides after mixing are two major issues in pesticide storage management. Thus, understanding the best storage condition for unused termiticides is vital to avoid termiticide degradation.

In Malaysia, pest control officers (PCOs) or field biologists commonly mix termiticides in polyethylene containers with varying sizes and colors. The containers are chosen as their durability is higher compared to glass containers. Meanwhile, the excess of mixed termiticides is placed inside a vehicle or in a store. It is widely known that photodegradation is an important factor influencing the degradation of pesticides (Martínez et al., 2009). Hence, uncovered containers placed in the vehicle might affect termiticides efficiency due to the exposure to sunlight. On a contrary, containers placed inside the store may have some protection from sunlight.

Studies on the stability of imidacloprid and fipronil in aqueous solution stored in polyethylene tanks have been done by previous studies (Spomer et al., 2009; Husen et al., 2009). They found that the tested termiticides were stable within the two weeks storage period. However, these studies were conducted in cooler regions where the environmental conditions were somewhat different from warmer regions. Thus, different exposure to sunlight and temperature might lead to different results.
In this study, three most commonly used termiticides i.e. bifenthrin, fipronil, and imidacloprid were evaluated for optimum storage conditions. Active ingredients (AI) of each termiticide were also examined using two different bottle colors (black and white) and different storage spaces (in car and store) up to 30 days

\section{MATERIALS AND METHODS}

\subsection{Preparation of termiticide solutions}

Termiticides containing three AIs i.e. fipronil (5.0\%), bifenthrin $(10.0 \%)$, and imidacloprid (20\%) were used in this study. The solutions were prepared by diluting the termiticides with distilled water and the concentration was standardized to $1000 \mathrm{ppm}$. Next, $500 \mathrm{ml}$ of the termiticide solutions were transferred into $500 \mathrm{ml}$ polyethylene bottles with two different colors i.e. white and black. The solutions were prepared in triplicate and were placed in the box (Figure 1).

\subsection{Study sites}

School of Biological Sciences, Universiti Sains Malaysia was selected as the study site. Two polyethylene bottles containing termiticides were assigned to two different locations i.e. in a chemical store and inside the back seat of the car (Naza Ria, Malaysia) (Figure 2 and 3). The car was left at the same location throughout the experiment. Then, $50 \mathrm{ml}$ of termiticide samples from each bottle were collected after 14 and 30 days. The $\mathrm{pH}$ value of each termiticide was measured using a $\mathrm{pH}$ meter (HANNA HI 8424, Romania). 


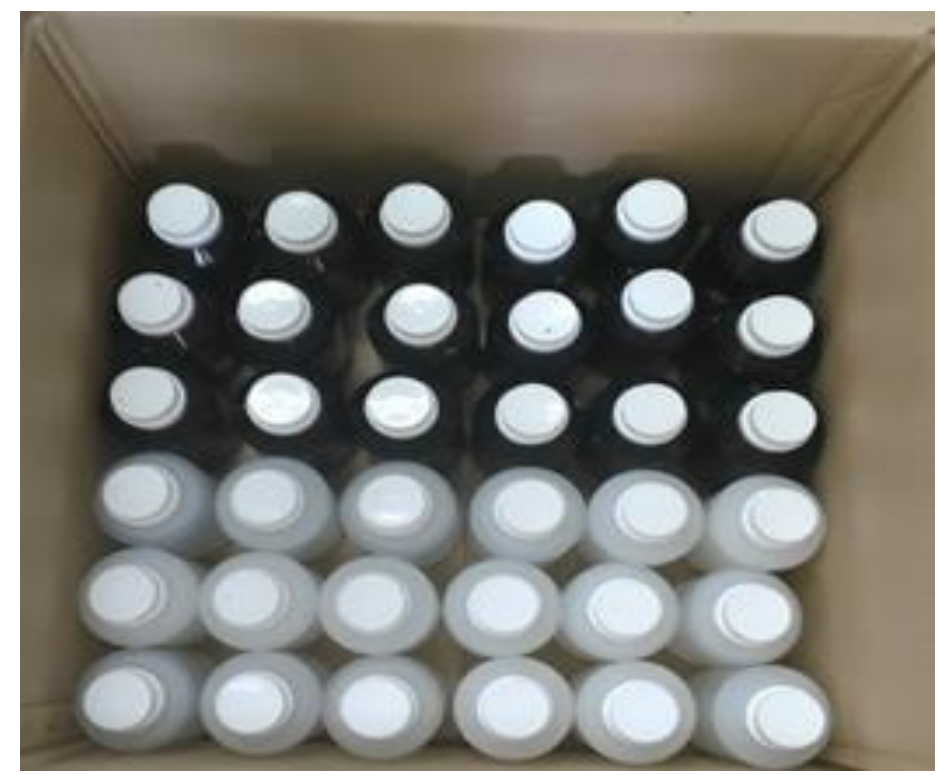

Figure 1. Polyethylene bottles setup inside the box

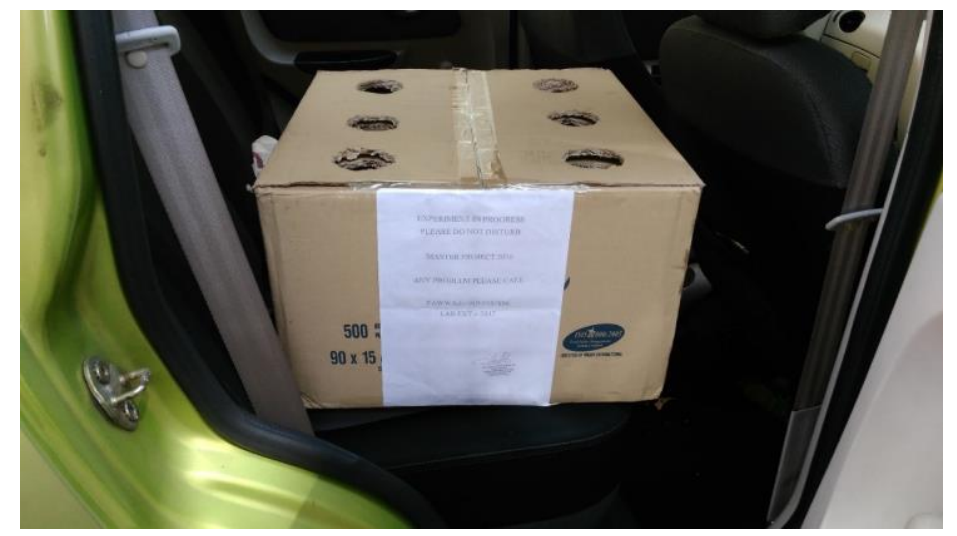

Figure 2. Polyethylene bottles place inside the car back seat

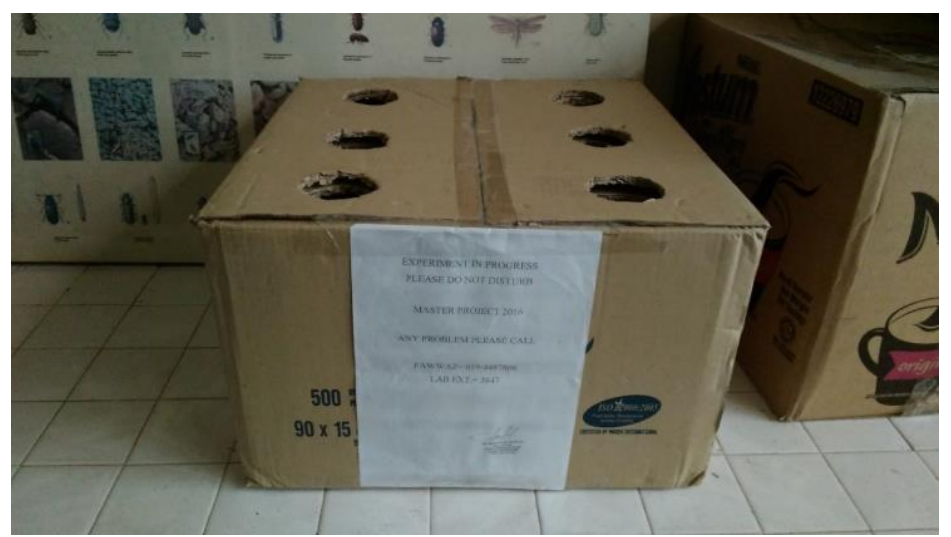

Figure 3. Polyethylene bottles in a chemical store 


\subsection{Sample extraction}

After the collection, $50 \mathrm{ml}$ of the termiticide samples were stored in a chest freezer (Hisense, HFZ-130D, Malaysia) at $16^{\circ} \mathrm{C}$ to prevent degradation until further use. About $10 \mathrm{ml}$ of the samples were mixed with $10 \mathrm{ml}$ of acetonitrile (ACN) in a universal bottle. The sample solution was vortexed for 10 seconds, was allowed to settle down for 30 minutes to obtain a supernatant and was later transferred into another universal bottle. About $10 \mathrm{ml}$ of $\mathrm{ACN}$ were added into the bottle. Next, $1 \mathrm{ml}$ of the supernatant was sonicated using an ultrasonic machine prior to HPLC analysis.

\subsection{Residual analysis}

In brief, the residual analysis was performed using HPLC (Agilent
Technologies, 1260 Infinity, Model number G1311C) coupled with photodiode array detector (PDA) (Agilent Technologies) as in Figure 4. The technical grade of bifenthrin (98.8\%), fipronil (97.9\%) (Sigma-Aldrich, Malaysia) and imidacloprid (99.5\%) (Chem Service) were used as standards. The standards and samples were dissolved using ACN (Baker, China). The HPLC-grade ACN (Baker) and ultra-pure water (Milipore Asia Limited, Selangor, Malaysia) were used as the mobile phase and were prepared at different ratios based on AIs.

The injection volume was set at 20.0 ul, passing through a single $\mathrm{C}_{18}$ column (4.6 $\mathrm{mm} \times 250 \mathrm{~mm}$ ) (Agilent Technologies, Eclipse Plus) with $5 \mu \mathrm{m}$ particle size. The flow rate was set at $1.0 \mathrm{ml} / \mathrm{min}$. Chromatographic conditions for all termiticides are summarized in Table 1.

Table 1. Chromatographic conditions for termiticide analyses

\begin{tabular}{lccc}
\hline Active ingredient (AI) & Water: ACN & Wavelength (nm) & $\begin{array}{c}\text { Retention time } \\
\text { (minutes) }\end{array}$ \\
\hline Bifenthrin & $5: 95$ & 204 & 6.24 \\
Fipronil & $30: 70$ & 280 & 6.72 \\
Imidacloprid & $70: 30$ & 270 & 5.95 \\
\hline
\end{tabular}

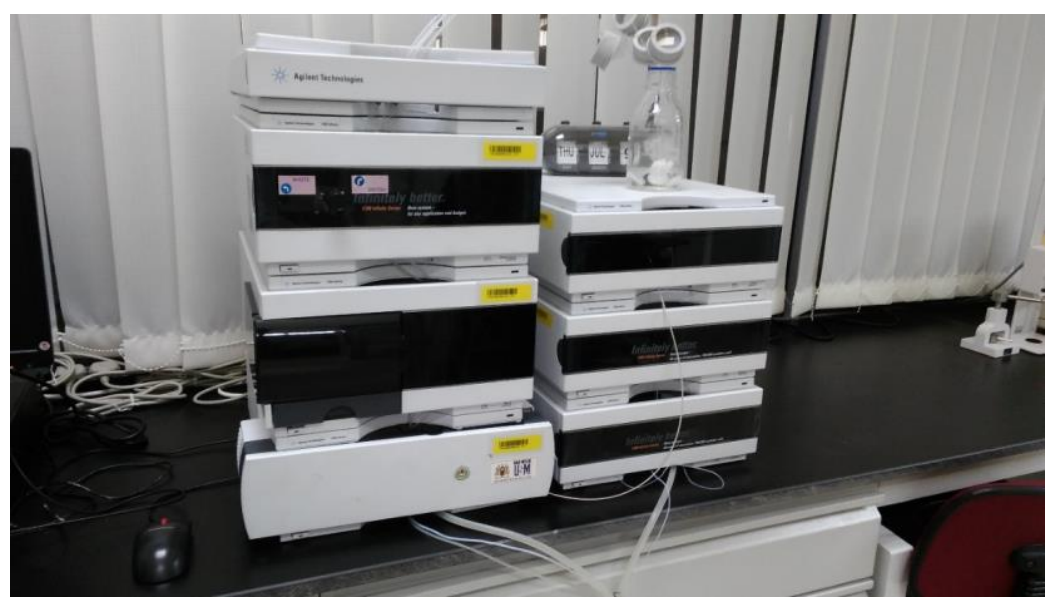

Figure 4. HPLC system used for termiticide analyses 


\subsection{Statistical analysis}

Concentrations of each termiticide

residue were determined using a standard formula regulated by CEPA, (1993) and Ong et al., (2016):

\section{$($ Spl H)(Std C)(Std V)(V) \\ $\mathbf{R}=$ \\ $(\operatorname{Std} \mathbf{H})(\mathbf{S p l}$ V)(Spl W)}

Where $\mathrm{R}$ is termiticide residue of part per million (ppm); $S p l H$ is the sample peak height area, Std $C$ is the standard concentration in ppm, Std $V$ is the standard volume injected in $\mu 1, V$ is the volume after the injection in $\mathrm{ml}, \mathrm{Std} H$ is the standard peak height area, $S p l V$ is the sample volume injected in $\mu 1$, and $S p l W$ is the sample weight in $\mathrm{g}$. The half-life of the termiticide residuals was calculated following a method described by Ong et al., (2016), whereas the degradation rate was calculated based on Lin et al. (2008) and Mahiudddin et al. (2014).

Statistical analysis was carried out using IBM SPSS Statistics version 21. Data transformation was done as the results were not normal. Analysis of variance (ANOVA) was performed to determine the significant differences between termiticides, bottle colors, and locations used in this study.

\section{RESULTS}

From the chromatographic analysis, each termiticide was detected at $6.72 \mathrm{~min}$ (fipronil), $5.95 \mathrm{~min}$ (imidacloprid) and 6.24 min (bifenthrin), respectively. Fipronil had the lowest residual; losing $99.53 \%$ (5.36 ppm) from its initial concentration after 30 days (Figure 5). Imidacloprid, on the other hand, exhibited the highest residual after 30 days with $110.05 \mathrm{ppm} \quad(88.80 \% \quad$ lost$)$. Degradation was also observed in bifenthrin where the concentration of this termiticide decreased to $60.93 \mathrm{ppm}$ (94.80\% lost) from the initial concentration. Evaluation of degradation rate for fipronil, bifenthrin, and imidacloprid, were $29.66,31.30$ and 33.16 $\mathrm{ppm} /$ day, respectively. Meanwhile, fipronil, bifenthrin, and imidacloprid had the halflives of 3.98, 7.45, and 9.42 days (Table 2). The $\mathrm{pH}$ values of fipronil, bifenthrin, and imidacloprid ranged from 4.58 to $5.12,4.22$ to 5.34 , and 4.45 to 5.05 , respectively. A survey done at 12 a.m. indicated that light intensity in the car was higher (1048-2600 lux) compared to in the store (28-37 lux). 


\section{Termiticide residual after 30 days}

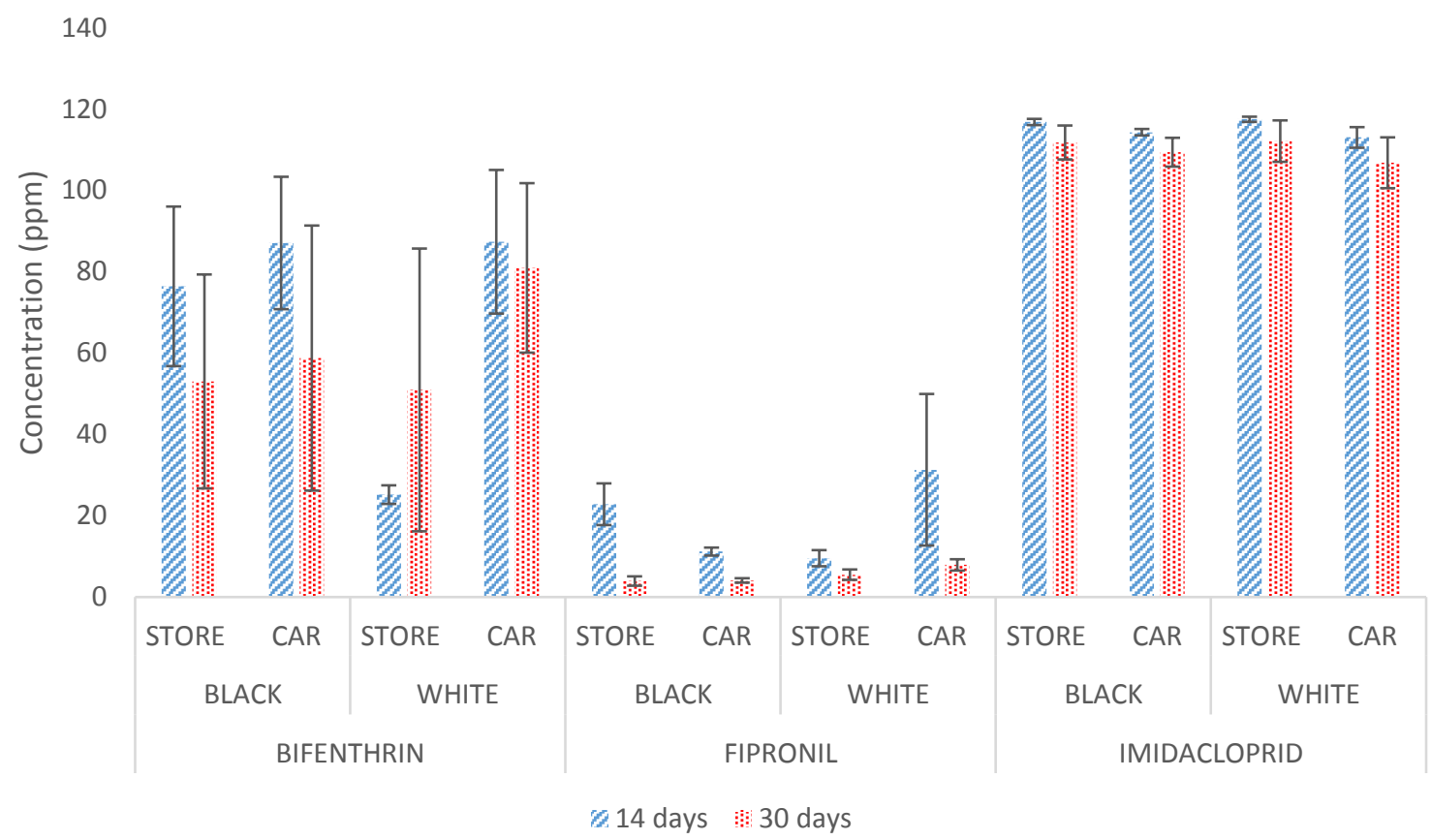

Figure 5. Concentrations of termiticides (bifenthrin, fipronil, and imidacloprid) after 30 days

Table 2. The degradation rate and half-life of bifenthrin, fipronil, and imidacloprid

\begin{tabular}{lcc}
\hline Termiticide & Rate of degradation (ppm/day) & Half-life (days) \\
\hline Bifenthrin & 31.30 & 7.45 \\
Fipronil & 33.16 & 3.98 \\
Imidacloprid & 29.66 & 9.42 \\
\hline
\end{tabular}

A two-way ANOVA analysis indicated that only termiticides showed a significant difference $(\mathrm{F}=154.112, \mathrm{df}=2, \mathrm{P}=$ 0.000). Other variables i.e. time interval $(\mathrm{F}=$ 1.265, $\mathrm{df}=2, \mathrm{P}=0.288)$, bottle colors $(\mathrm{F}=$ $0.181, \mathrm{df}=1, \mathrm{P}=0.672)$ and places $(\mathrm{F}=5.495$, $\mathrm{df}=1, \mathrm{P}=0.977$ ) had no significant effecton concentrations of the tested termiticides. The combined variables also had no significant effect on termiticide concentrations (Table 3). 
Table 3. Effects of the month, termiticide, color, and place on termiticide concentrations

\begin{tabular}{|c|c|c|c|c|}
\hline Source & df & Mean square & $\begin{array}{c}\text { F- } \\
\text { value }\end{array}$ & $\begin{array}{c}\text { P- } \\
\text { value }\end{array}$ \\
\hline MONTH & 1 & 1442.114 & 2.377 & 0.13 \\
\hline TERMITICIDE & 2 & 60901.928 & 100.388 & $\mathbf{0}$ \\
\hline COLOR & 1 & 57.835 & 0.095 & 0.759 \\
\hline PLACE & 1 & 1419.646 & 2.34 & 0.133 \\
\hline MONTH * TERMITICIDE & 2 & 98.704 & 0.163 & 0.85 \\
\hline MONTH $*$ COLOR & 1 & 576.584 & 0.95 & 0.335 \\
\hline MONTH $*$ PLACE & 1 & 257.38 & 0.424 & 0.518 \\
\hline TERMITICIDE $*$ COLOR & 2 & 179.176 & 0.295 & 0.746 \\
\hline TERMITICIDE * PLACE & 2 & 1572.572 & 2.592 & 0.085 \\
\hline COLOR $*$ PLACE & 1 & 1419.113 & 2.339 & 0.133 \\
\hline MONTH $*$ TERMITICIDE $*$ COLOR & 2 & 658.515 & 1.085 & 0.346 \\
\hline MONTH $*$ TERMITICIDE * PLACE & 2 & 140.1 & 0.231 & 0.795 \\
\hline MONTH $*$ COLOR $*$ PLACE & 1 & 440.6 & 0.726 & 0.398 \\
\hline TERMITICIDE $*$ COLOR $*$ PLACE & 2 & 610.45 & 1.006 & 0.373 \\
\hline MONTH * TERMITICIDE * COLOR * PLACE & 2 & 100.925 & 0.166 & 0.847 \\
\hline
\end{tabular}

df, degree of freedom; significant values are given in bold

Concentrations of termiticides (ppm) placed in the store for 30 days are illustrated in Figure 6. Imidacloprid showed the highest concentration (111.98 ppm), followed by bifenthrin (51.99 $\mathrm{ppm})$, and fipronil (4.72 ppm).

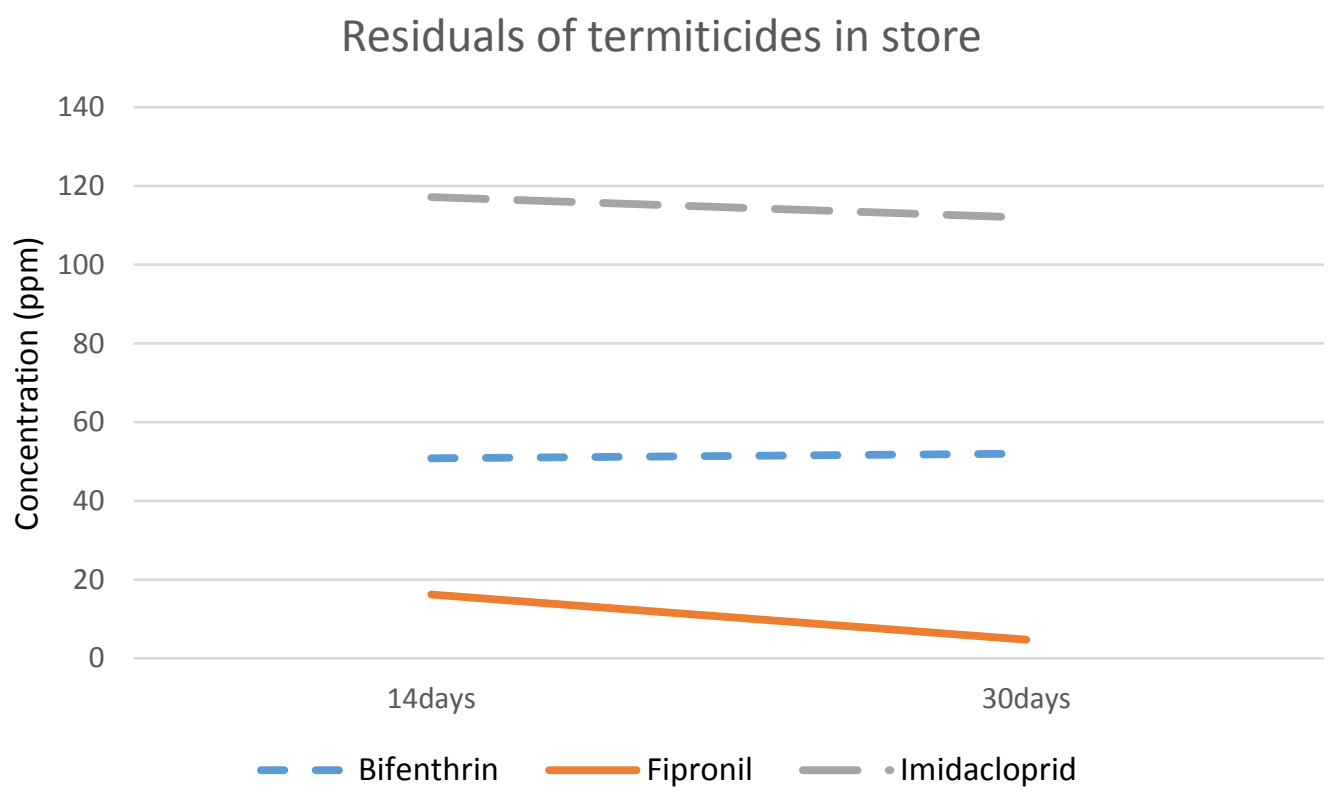

Figure 6. Residuals of bifenthrin, fipronil and imidacloprid placed in the store for 30 days

A continual decrease of concentrations was also noted in termiticides placed in the car (Figure 7). The decrease of fipronil concentration was the highest i.e. $5.99 \mathrm{ppm}$, followed by bifenthrin (69.87 ppm), and imidacloprid (108.12 ppm). 


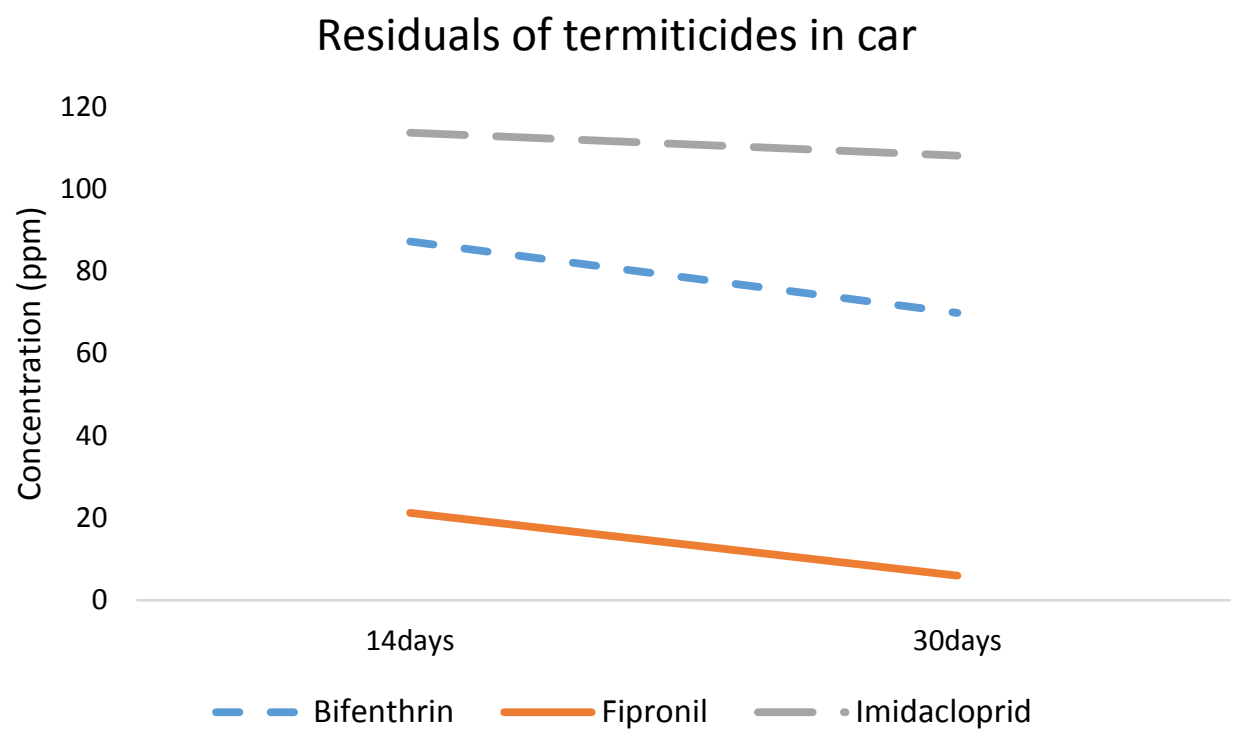

Figure 7. Residuals of bifenthrin, fipronil, and imidacloprid placed in the car for 30 days

\section{DISCUSSIONS}

All termiticides had a great loss, ranging from $88.80 \%$ to $99.53 \%$ from their initial concentrations, corroborating the fact that these termiticides were unstable in aqueous solution after 30 days. The residual of imidacloprid was high compared to bifenthrin and fipronil after 30 days. However, a greater loss was noted in imidacloprid compared to bifenthrin and fipronil. Hydrolysis might be one of the contributing factors in the major dissipation of these termiticides. To make matter worse, the chemical properties of termiticides, $\mathrm{pH}$ and temperature could further increase the rate of hydrolysis.

Hydrolysis half-life of imidacloprid is 30 days $\left(25^{\circ} \mathrm{C}, \mathrm{pH} 7\right)$ (Fossen, 2006). Hydrolysis of this termiticide was said to be rapid under basic condition but slower in acidic and neutral water (Zheng \& Liu, 1999). Their study was well-supported by Liu et al. (2006) of which hydrolysis of imidacloprid was more rapid under alkaline condition compared to acidic and neutral conditions. Contrasted to the abovementioned studies, Sarkar et al. (1999), however, found no significant difference in the hydrolysis of imidacloprid either under acidic, alkaline or neutral conditions. In this study, imidacloprid experienced a high rate of degradation although the $\mathrm{pH}$ values were considered as acidic (4.45 to 5.05). The temperature was presumably to be the major factor for imidacloprid hydrolysis (Zheng \& Liu, 1999). They reported that the hydrolysis of imidacloprid was temperature-dependent of which the rate of the hydrolysis increased with the increase of temperature.

As with bifenthrin, the occurrence of hydrolysis is currently unknown as no study was done so far. Fecko (1999) claimed that bifenthrin was considered stable. But this proclamation was not supported by Floate et al. (2005). They indicated that hydrolysis of deltamethrin, a termiticide that belongs to a similar group of bifenthrin did occur where the half-life was 2.5 days $\left(\mathrm{pH} 9,25^{\circ} \mathrm{C}\right)$.

Thus, based on the finding of Floate et al. (2005), it can be assumed that bifenthrin 
is unstable in aqueous and undergoes hydrolysis as well. Furthermore, the result indicated bifenthrin degraded rapidly due to photodegradation. It was supported by Tariq et al. (2017), where they found that bifenthrin and deltamethrin underwent a rapid degradation due to UV irradiation. After 24 hours, about 41 to $57 \%$ of bifenthrin degraded after being exposed to UV irradiation. Thus, it was possible that bifenthrin was highly dissipated after 30 days of storage. Up to now, studies on photodegradation of bifenthrin is limited and scarcely discussed. Deltamethrin that belongs to the same group with bifenthrin was almost degraded completely after being exposed to UV irradiation after 160 seconds (Liu et al., 2010). Therefore, it can be suggested that bifenthrin is also unstable under the UV light since it belongs to the same group as deltamethrin. Generally, light has a tendency to absorb, refract, reflect or transmit when in contact with an object. A white object is able to reflect all light wavelength, while black object absorbs all light wavelength. However, no significant difference was observed between different bottle colors used in this study

A 14-days experiment on fipronil showed that this termiticide was stable in slightly acidic to neutral solutions without light and might also be stable during hydrolysis (Husen et al., 2009). A study by Connelly (2001) found that the hydrolysis half-lives of fipronil were 11.3 days and 15.6 days when stored at $32{ }^{\circ} \mathrm{C}$ under basic $(\mathrm{pH}$ 9.1) and neutral ( $\mathrm{pH}$ 7.1) conditions. Meanwhile, a previous study demonstrated that the concentrations of fipronil reduced to $80 \%$ in acidic, $92 \%$ in neutral and $98 \%$ in basic buffers when stored up to 60 days (Ramesh \& Balasubramanian, 1999).

The vulnerability of polyethylene bottles to sunlight was higher when placed in the car compared to those of in the store. One of the contributing factors to termiticide dissipation is photodegradation. This degradation rate is also highly influenced by temperature. For example, the photodegradation half-life of imidacloprid was $8.5 \mathrm{~min}$ at $29.85^{\circ} \mathrm{C}$ and was further decreased to $7.8 \mathrm{~min}$ at $39.85^{\circ} \mathrm{C}$ (Liu et al., 2006). Imidacloprid also has been reported to experience rapid photolysis under UV irradiation (Wamhoff \& Schneider, 1999). Based on these studies, it thus can be concluded that imidacloprid degrades rapidly although the exposure to sunlight within a short period of time. In the same work, Wamhoff \& Schneider (1999) stated that in a presence of UV light, the photolysis half-life of imidacloprid in pure water was $46 \mathrm{~min}$, indicating that imidacloprid was also unstable in pure water and was easily degraded.

Fipronil is photostable in water for up to two weeks (Husen et al., 2009). It can be observed in this study where its concentration was insignificant either under the sunlight or shaded area. However, fipronil was found to be unstable when the storage period was prolonged up to 30 days which was probably due to photolysis

To date, existing knowledge on photodegradation of bifenthrin is scarce and largely limited. Deltamethrin, a termiticide that belongs to the same group with bifenthrin almost fully degraded after being exposed to UV irradiation within $3 \mathrm{~min}$ (Liu et al., 2010). Therefore, a similar assumption can be made with bifenthrin. Adding to this, results from this study also showed that the photodegradation of bifenthrin was also rapid. Tariq et al. (2017) also stated that bifenthrin and deltamethrin underwent a rapid degradation due to UV irradiation. Bifenthrin degraded approximately 41 to $57 \%$ after being exposed to UV irradiation 
for 24 hours. Thus, high dissipation of bifenthrin may occur after 30 days of storage. The statistical analysis revealed that there was no significant difference between different bottle colors used in this study. A previous study by Spomer et al. (2009) also found the concentrations of imidacloprid in a polyethylene tank had no significant difference with that of in a glass jar. Its concentrations remained unchanged although light penetrated the polythene tank and the glass jar in a different manner. The result was somewhat similar concerning light penetration between black and white bottles used in this study because there is no significance difference between the bottle color.

The survey done at 12 p.m indicated that light penetration was higher in the car (1048-2600 lux) compared to in the store (2837 lux). However, no significant difference was observed between termiticides stored at these two locations on the termiticide concentration degradation. This result fits well with the previous finding by Husen et al. (2009) of which they reported no significant difference between termiticides placed under direct sunlight and shaded areas.

\section{CONCLUSIONS}

In conclusion, all termiticides lost $88.80 \%$ to $99.53 \%$ from their initial concentrations, suggesting that they were unstable in aqueous solution after 30 days. These termiticides were also less effective after 14 days after mixing. The study also found that the bottle colors and storage conditions exhibited no significant effect on the termiticides concentrations. The Pest Control Operator (PCO) are advised not to used left over mix termiticides or insecticides after 14 days to provide effective termite pest management.

\section{ACKNOWLEDGEMENTS}

The project was granted under (USM) Research University Grant (RU) 1001 / PBIOLOGI / 811241.

\section{REFERENCES}

Connelly, P. (2001). Environmental fate of fipronil. Environmental Monitoring Branch. Department of Pesticide Regulation. California Environmental Protection Agency, Sacramento, CA, 1 - 17Fecko, A. (1999). Environmental fate of bifenthrin. Environmental Monitoring and Pest Management Branch. California Department of Pesticide Regulation.

Floate, K. D., Wardhaugh, K. G., Boxall, A. B. A., and Sherratt, T. N. (2005). Fecal Residues of Veterinary Parasiticides: Nontarget Effects in the Pasture Environment. Annual Review of Entomology, 50(1), 153-179.

Fossen, M. (2006). Environmental Fate of Imidacloprid. Department of Pesticide Regulation.

Husen, T. J., Spomer, N. A., and Kamble, S. T. (2009). Degradation of fipronil (termidor) in aqueous solution stored in polyethylene tanks exposed to sunlight or shade. Bulletin of Environmental Contamination and Toxicology, 82(5), 605-609.

Joshi, V., Srivastava, A., and Srivastava, P. C. (2016). Potential of some soil amendments in reducing leaching of fipronil to groundwater. International Journal of Environmental Science and Technology, 13(2), 631-638. 
Liu, P., Liu, Y., Liu, Q., and Liu, J. (2010). Photodegradation mechanism of deltamethrin and fenvalerate. Journal of Environmental Sciences, 22(7), 11231128.

Liu, W., Zheng, W., Ma, Y., and Liu, K. (2006). Sorption and degradation of imidacloprid in soil and water. Journal of Environmental Science and Health Part B Pesticides, Food Contaminants, and Agricultural Wastes, 41(5), 623634.

Mahiudddin, M., N. M. Fakhruddin, A. M. Abdullah, M.A.Z. Chowdhury, M. A. Rahman, and M. K. Alam. 2014. Degradation of the organophosphorus insecticide diazinon by soil bacterial isolate. Int. J. Biotechnol. 3: 12-23.

Martínez Vidal, J. L., Plaza-Bolaños, P., Romero-González, R., and Garrido Frenich, A. (2009). Determination of pesticide transformation products: A review of extraction and detection methods. Journal of Chromatography A, 1216(40), 6767-6788.

Ong, S. Q., Ab Majid, A. H., \& Ahmad, H. (2016). Degradation of insecticides in poultry manure: Determining the insecticidal treatment interval for managing house fly (Diptera: Muscidae) populations in poultry farms. Journal of Economic Entomology, 109(2), 952957.

Pesticide Act. (1974). Law of Malaysia: Act 149 PESTICIDES ACT 1974.

Petroff, R. (2010). Safe Handling of Pesticides - Mixing. Montana State University.
Phung, D. T., Connell, D., Miller, G., and Chu, C. (2012). Pesticide regulations and farm worker safety: the need to improve pesticide regulations in Viet Nam. Bulletin of the World Health Organization, (90), 468-473.

Ramesh, A., and Balasubramanian, M. (1999). Kinetics and hydrolysis of fenamiphos, fipronil, and trifluralin in aqueous buffer solutions. Journal of Agricultural and Food Chemistry, 47(8), 3367-3371.

Sarkar, M. A., Biswas, P. K., Roy, S., Kole, R. K., and Chowdhury, A. (1999). Effect of $\mathrm{pH}$ and type of formulation on the persistence of imidacloprid in water. Bulletin of Environmental Contamination and Toxicology, 63(5), 604-609.

Spomer, N. A., Husen, T. J., and Kamble, S. T. (2009). Stability of imidacloprid (Premise 75) in a tank-mixed aqueous solution when stored in shade or sunlight. Bulletin of Environmental Contamination and Toxicology, 82(1), 116-119.

Tariq, S. R., Ahmed, D., Farooq, A., Rasheed, S., and Mansoor, M. (2017). Photodegradation of bifenthrin and deltamethrin - effect of copper amendment and solvent system. Environmental Monitoring and Assessment, 189(2), 1-9.

Transporting and Storing Pesticides Safely. (2016). University of Kentucky Department of Entomology.

Wamhoff, H., and Schneider, V. (1999). Photodegradation of imidacloprid. Journal of Agricultural and Food Chemistry, 47, 1730-1734. 
Zheng, W., and Liu, W. (1999). Kinetics and mechanism of the hydrolysis of imidacloprid. Pesticide Science, 55(4), 482-485. 